\title{
Soft-Embalmed Surgical Donors as a Teaching Model for Simulated Orthopedic Skills
}

\author{
Abercrombie C, Becker R, Roepke WB, Serbin R, Newby J, Lance D, Harris E and Kwasigroch T* \\ Department of Orthopedic, USA.
}

*Corresponding author: Kwasigroc T, Department of Orthopedic, USA.

\begin{abstract}
Soft-embalmed, surgical donors are a valuable resource for medical professionals to utilize in low stress environments. Simulated procedural workshops can be used to establish competence and improve confidence for a variety of orthopedic skills using this life-like teaching model. The authors propose that soft-embalmed, surgical donors are suitable training models for teaching these procedural skills (new, updated guidelines, or those rarely used). We believe that their incorporation into medical school curriculums and continuing education sessions will result with improvements in confidence and competence in performing the associated procedural skill.
\end{abstract}

Keywords: Experiential learning; Hip injection; Knee arthrocentesis; Simulation; Skill workshop; Soft-embalm; Thiel Embalming

\section{Introduction}

Performance in invasive skills can rapidly improve with just a single cadaver laboratory session and skills learned these sessions can be translated to and sustained in the clinical setting to produces competent providers who perform skills rapidly and with minimal complications [1]. Many procedures are performed at low frequency, and providers do not have the opportunity to practice on a life-like model outside of clinical settings [2]. For example, \ultrasound-guided hip injections minimize risk in damaging adjacent neurovascular structures. A lack of ultrasound guided training is a hindrance to widespread use [3]. In addition, medical students are frequently exposed to procedures such as knee arthrocentesis during their clinical rotations, but there is no suitable model for medical students to practice performing the procedure or interpret the results. Simulated patients cannot be utilized due to the invasive nature of the procedure, and formalinembalmed cadavers are unable to provide a realistic experiential learning opportunity due to the postmortem rigidity and high susceptibility to tissue decay. The absence of a sufficient teaching model often results in procedures being done for the first time, or for the first time in an extended period of time, on actual patients.

We believe that the use of soft-embalmed, surgical donors can provide a solution to this gap in medical education. The surgical donors are prepared using a modified Thiel embalming method. In contrast to traditional formalin-embalmed cadavers, soft-embalming allows for increased, life-like joint mobility and preserved fascial integrity while closely mimicking the aesthetics of live tissue $[4,5]$. These unique qualities allow skills practice in a realistic environment. Cadavers prepared with embalming methods that provide more realistic joint motion and visual and tactile tissue fidelity are significantly superior to formaldehyde fixed donors for surgical skills training are a significant improvement for high-fidelity orthopedic surgical skills training and surgical skills training $[6,7]$. We have developed a variety of surgical donor skills workshops for learners and providers at various levels of 
training. These workshops include lumbar puncture, central line cannulation, airway management, paracentesis, thoracentesis, and upper extremity injections, knee arthrocentesis, and hip injections.

\section{Study Analysis}

During lower extremity joint workshops, participants completed a pre-survey and post-survey to assess perceived confidence, experience and ability to perform the procedure. Participants were informed of the knowledge and the steps required for mastery of the skill before each teaching encounter. The anatomy and the potential complications of each procedure were reviewed. Individual attention was given to participants as needed. Skills were repeated until they were performed correctly because the goal was mastery of the skill. Faculty served as both instructors and evaluators. Each learner was assessed using a validated competency checklist.

The surveys asked about: 1 . overall quality of the experiences, 2. if the session improved their confidence in performing the skill, 3. if the setting improved their ability to properly perform the skill in an actual patient care setting, 4. if they believed simulated practice using surgical donors to be an effective method for learning procedural skills, and 5. if they preferred the use of soft-embalmed, surgical donors over formalin-embalmed cadavers as a teaching tool.

Sixty-eight medical students completed a knee arthrocentesis workshop. $96 \%$ of participants reported in the post-survey they felt confident performing knee arthrocentesis under physician supervision following their participation in the laboratory session (versus $15 \%$ of participants in the pre-survey as seen in figure 1). $96 \%$ of participants reported that the soft-embalmed, surgical donors provided a more realistic teaching model than formalinembalmed for learning knee arthrocentesis (figure 2). 100\% of participants believed the incorporation of simulated practice using soft-embalmed donors an effective method in teaching students to perform knee arthrocentesis (figure 3). 100\% of participants reported that they would participate in future sessions using this model to learn and practice other orthopedic procedural techniques. This study used a moderate sample size of thirdyear medical students to provide data regarding the suitability of using soft-embalmed, donors in arthrocentesis education. Results indicate they are effective tools in teaching medical students to perform knee arthrocentesis, that students preferred the model to the formalin cadavers, and that the model is a safe, engaging, and high-quality teaching modality for demonstrating proper arthrocentesis procedural technique to medical students.

\section{I feel confident performing knee arthrocentesis under physician supervision.}

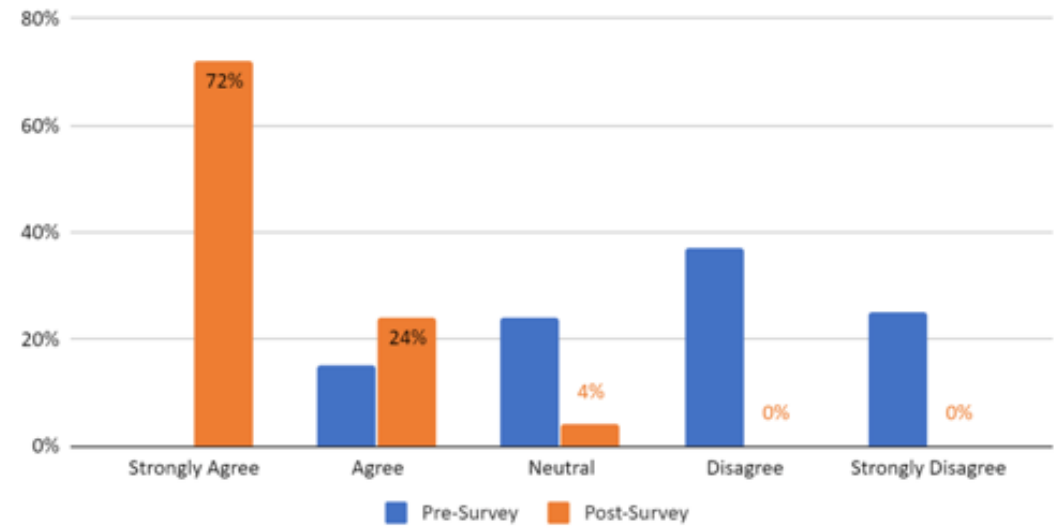

Figure 1: Displays the differences between the participants' perceived confidence in performing knee arthrocentesis under physician supervision before and after participating in this study. In the pre-survey, ten individuals (15\%) reported that they felt confident performing knee arthrocentesis under physician supervision ( $0 \%$ strongly agree, $15 \%$ agree). In the post-survey, sixty-five students $(96 \%)$ reported that they felt confident performing knee arthrocentesis under physician supervision.

Health care providers completed a hip injection workshop as a part of a continuing education series. The results from the hip injection pre- and post-survey Likert scales showed 100\% of participants found the simulation valid, would transfer to patient care and improve their identification of anatomical landmarks using ultrasound. The majority of participants felt more prepared (78\%) and confident (89\%) to perform ultrasound-guided hip injections following the simulation and preferred learning with a didactic and surgical donor. Lastly, $100 \%$ of participants successfully demonstrated competency through checklist evaluation. We expect future studies to show an improvement in skill performance. This study used a small sample size (9 participants) of practicing providers to provide data regarding the suitability of using softembalmed, surgical donors in hip injection training. Results indicate 
the surgical donor is an effective teaching model, participants preferred the model to the formalin donors, and the model is a highquality teaching modality for demonstrating proper hip injection procedural skills.

\section{Perspective}

In conclusion, soft-embalmed, surgical donors are an effective educational tool in teaching medical students to perform knee arthrocentesis and an effective tool in teaching providers ultrasound guided hip injections. The soft-embalmed, surgical donor model is felt to be a more suitable teaching model to formalin-embalmed donors for arthrocentesis and hip injection simulated procedures and this model potentially generalizable to the instruction of a variety of orthopedic procedures. Further research considerations include the evaluation of patient outcomes in clinical practice relative to the procedure, including accuracy of injection location, post-procedure infection rates due to improper sterile technique, and patient satisfaction relative to the procedure (Figures 1-3).

The soft-embalmed donor provided a more realistic training model than the formalin-embalmed donor.

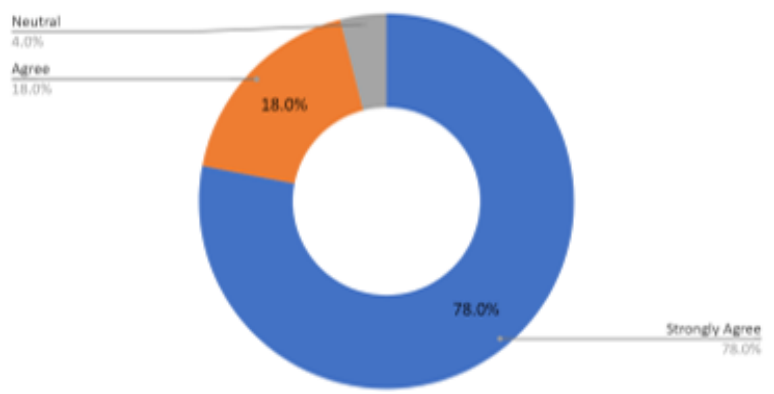

Figure 2: Displays the participants' perceptions of whether the soft-embalmed cadavers or formalin-embalmed cadavers provided a more realistic training model when learning to perform knee arthrocentesis. Sixty-five individuals $(96 \%)$ reported feeling that the soft-embalmed cadavers provided a more realistic teaching model than formalin-embalmed cadavers for learning knee arthrocentesis $(78 \%$ strongly agree, $18 \%$ agree, $4 \%$ neutral)

\section{I believe the utilization of soft-embalmed donors is an effective method for teaching orthopedic procedural skills.}

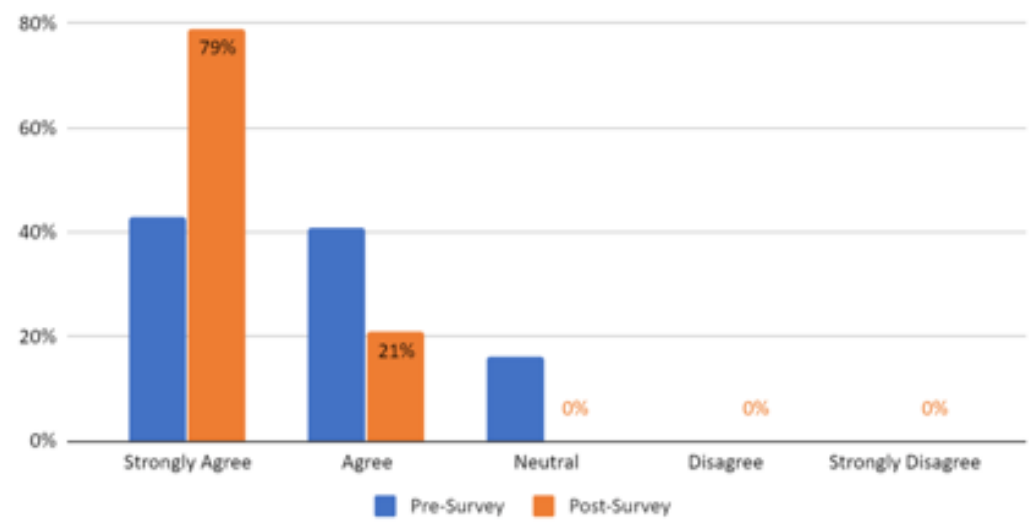

Figure 3: Displays the pre-survey and post-survey differences regarding the participants' perceived effectiveness of soft-embalmed as an educational tool when teaching orthopedic procedural skills such as knee arthrocentesis. In the pre-survey, fifty-seven students (84\%) reported that they believed the incorporation of simulated practice using soft-embalmed cadavers is an effective method for teaching orthopedic procedures such as knee arthrocentesis ( $43 \%$ strongly agree, $41 \%$ agree). In the post-survey, all sixty-eight individuals reported that they believed the incorporation of simulated practice using soft-embalmed cadavers is an effective method for teaching orthopedic procedures such as knee arthrocentesis ( $79 \%$ strongly agree, $21 \%$ agree).

\section{Acknowledgement}

None.

\section{Conflict of Interest Statement}

The authors have no conflict of interest to declare.

\section{References}

1. Martin M, Vashisht B, Frezza E, Ferone T, Lopez B, Pahuja, et al. (1998) Competency-based instruction in critical invasive skills improves both resident performance and patient safety. Surgery 124(2): 313-317.

2. Joy J, McLeod G, Lee N, Munirama S, Corner G, et al. (2015) Quantitative assessment of Thiel soft-embalmed human cadavers using shear wave elastography. Annals of Anatomy 202: 52-56. 
3. Payne JM (2016) Ultrasound-Guided Hip Procedures. Physical Medicine and Rehabilitation Clinics of North America 27(3): 607-629.

4. Ottone NE, Vargas CA, Fuentes R, Delsol M (2016) Walter Thiel's embalming method. Review of solutions and applications in different fields of biomedical research. Int J Morphol 34(4): 1442-1454.

5. Yiasemidou M, Roberts D, Glassman D, James Tomlinson, Shekhar Biyani, et al. (2017) A Multispecialty Evaluation of Thiel Cadavers for Surgical Training. World J Surg 41: 1201-1207.
6. Burns DM, Bell I, Katchky R, Dwyer T, Toor J, et al. (2018) Saturated Salt Solution Cadaver-Embalming Method Improves Orthopaedic Surgical Skills Training. The Journal of bone and joint surgery 100(15): e104.

7. Hayashi S, Homma H, Naito M, Oda J, Nishiyama T, et al. (2014) Saturated salt solution method: a useful cadaver embalming for surgical skills training. Medicine 93(27): e196. 